\title{
Muscle metabolic status in patients with severe COPD with and without long-term prednisolone
}

\author{
E.M. Pouw*, E. Koerts-de Lang*, H.R. Gosker*, G. Freling ${ }^{\#}$, G.J. van der Vusse ${ }^{+}$, \\ E.F.M. Wouters*, A.M.W.J. Schols**
}

Muscle metabolic status in patients with severe COPD with and without long-term prednisolone. E.M. Pouw, E. Koerts-de Lang, H.R. Gosker, G. Freling, G.J. van der Vusse, E.F.M. Wouters, A.M.W.J. Schols. C ERS Journals Ltd 2000.

ABSTRACT: Both abnormalities in high energy phosphate metabolism and a decreased oxidative enzyme capacity have been reported in skeletal muscle of stable chronic obstructive pulmonary disease (COPD) patients. The first aim of this study was to investigate whether these findings are present in anterior tibialis muscle and whether or not they are associated. Abnormalities in mitochondrial structure and function as well as signs of myopathy have been found during corticosteroid treatment. The second aim of this study, therefore, was to investigate whether in COPD patients prolonged use of low dose prednisolone has effects on muscle energy metabolism and qualitative morphology.

In a cross-sectional study 15 COPD patients (forced expiratory volume in one second (FEV1) $33 \pm 9$ (mean \pm SD) \% predicted) who were steroid-naive (CORT-) were compared with 10 healthy control subjects (HC) and with 14 COPD patients (FEV1 $30 \pm 11 \%$ pred), who had been using oral prednisolone for at least 1 yr (CORT + ).

It was found that adenosine triphosphate (ATP)/adenosine diphosphate was lower in CORT- compared to HC (5.7 versus 6.2, $\mathrm{p}=0.03)$. Inosine monophosphate was detected in 13 of 15 CORT- compared to 3 of $10 \mathrm{HC}(p=0.004)$. However, although indications were found for an imbalance in production and utilization of ATP, comparing CORT- and HC, no differences in oxidative (citrate synthase and 3-hydroxy-acylcoenzyme A dehydrogenase) and glycolytic (hexokinase, lactate dehydrogenase and phosphofructokinase) enzyme capacities were found.

When, comparing steroid-treated and steroid-naive patient subgroups, no differences in the above mentioned parameters of muscle energy metabolism and of muscle qualitative morphology were found.

Eur Respir J 2000; 16: 247-252.

Exercise intolerance and dyspnoea are the most frequently occurring complaints in patients with severe chronic obstructive pulmonary disease (COPD). Weakness of both skeletal and respiratory muscles contributes significantly to these complaints [1]. Recently it has been shown that muscle weakness in COPD patients can predominantly be explained by muscle atrophy [2]. However, several studies suggested that exercise tolerance might also be impaired by alterations in muscle energy metabolism [3-5].

Experimental evidence is accumulating that in COPD patients muscle energy metabolism is already disturbed at rest. JAKOBSSON et al. [6] found a decreased oxidative capacity and an increased glycolytic capacity in quadriceps femoris muscle. Maltais et al. [3] reported a decreased oxidative capacity in quadriceps femoris muscle. In another recent study [4], in tibialis anterior muscle, indications for an imbalance in adenosine triphosphate (ATP) utilization and resynthesis were found, as suggested by increased inosine monophosphate (IMP) levels, which were negatively related with ATP/adenosine diphosphate (ADP) ratios. However, in that study oxidative and glycolytic enzyme capacities were not investigated.
Depts of *Pulmonology, ${ }^{+}$Physiology, and \#Pathology, Maastricht University, Maastricht, the Netherlands. *Asthmacentre Hornerheide, Horn, the Netherlands.

Correspondence: A.M.W.J. Schols Dept of Pulmonology

University Hospital Maastricht PO Box 5800 6202 AZ Maastricht

The Netherlands Fax: 31433875051

Keywords: Adenine nucleotide biochemistry

chronic obstructive pulmonary disease corticosteroids

myopathy

skeletal muscle

Received: June 201999

Accepted after revision March 132000

Supported by a scholarship from ASTRA BV, the Netherlands.
In view of the above mentioned findings in skeletal muscle of COPD patients, it might be hypothesized that the observed disturbances in high energy phosphate metabolism in the tibialis anterior muscle are associated with decreased oxidative enzyme capacities. Therefore, the first aim of the current study was to investigate both high energy phosphates and metabolic enzyme capacities in anterior tibialis muscle comparing stable severe COPD patients with healthy control subjects.

In experimental animal studies indications have been found that glucocorticosteroids may affect muscle energy metabolism. Several studies found alterations in muscular mitochondrial structure, including enlargement [7], aggregation and vacuolation [7, 8] of mitochondria. Furthermore, indications for impaired muscle glycogenolysis [9] and increased glycogen synthesis $[10,11]$ have been provided. However, virtually no information is available regarding muscle energy metabolism in humans using long-term corticosteroid therapy.

In a recent human study histological abnormalities were observed in biopsies of the quadriceps femoris muscle of COPD patients with severe steroid-induced myopathy 
[12]. Alterations in qualitative morphology were found, including increased variation of fibre diameters, increased numbers of central nuclei and increased amounts of connective tissue in between muscle fibres. In the above mentioned study the doses of corticosteroids administered were quite high. No studies are available investigating muscle morphology in COPD patients using long-term low dose corticosteroids. It might be hypothesized that treatment with long-term low dose prednisolone leads to altered muscle enzyme capacities, increased glycogen levels and increased occurrence of myopathic features. Therefore, the second aim of this study was to investigate parameters of muscle energy metabolism and muscle qualitative morphology comparing stable severe COPD patients who never received maintenance treatment with oral glucocorticosteroids with COPD patients using low dose oral prednisolone for $>1 \mathrm{yr}$.

\section{Methods}

\section{Subjects}

Fifteen COPD patients who never received maintenance treatment with oral corticosteroids (CORT-) were compared with 10 healthy age-matched volunteers (HC). CORTpatients were also compared with 14 COPD patients who had been using oral prednisolone for at least $1 \mathrm{yr}(\mathrm{CORT}+)$. None of the patients received steroid burst regimens within 3 months prior to the study. Part of the data was reported in an earlier study [4], involving a larger patient group. All patients had COPD [13] and had a forced expiratory volume in one second (FEV1) expressed as a percentage of predicted of $<50 \%$. Exclusion criteria were a history of cardiac failure, distal arteriopathy, malignancy, endocrine, hepatic or renal disease or use of anticoagulant drugs. All patients were clinically stable at the time of the study. Written informed consent was obtained and the study was approved by the Medical Ethical Board of the University Hospital Maastricht (the Netherlands).

\section{Corticosteroid and concomitant treatment}

Daily dose and duration of prednisolone treatment were obtained using a standardized patient questionnaire. The number of steroid burst regimens used in the year prior to examination was noted. All data were checked in available hospital files. COPD patients were treated with inhaled $\beta_{2-}$ agonists $(n=29)$, inhaled anticholinergics $(n=24)$, inhaled corticosteroids $(n=25)$, and oral theophylline $(n=22)$. None of the patients were using $>1,200 \mu \mathrm{g}$ inhaled steroids per day.

\section{Collection and analysis of muscle biopsies}

After an overnight fast, muscle biopsies were obtained under resting conditions, while the subjects were breathing room air. Under local anaesthesia, biopsies were taken from anterior tibialis muscle, using a conchotome [14]. Samples used for the determination of parameters of muscle energy metabolism were immediately frozen in liquid nitrogen and stored at $-80^{\circ} \mathrm{C}$. The average weight of individual muscle samples was (mean $\pm \mathrm{SD}) 26 \pm 5 \mathrm{mg}$. After freezedrying adherent blood and connective tissue were removed. ATP, ADP, adenosine monophosphate (AMP) and IMP were determined with a high performance liquid chromatographic technique [15]. The detection level for IMP was $0.06 \mathrm{mmol} \cdot \mathrm{kg}^{-1}$ dry weight. Phosphocreatine (PC) and creatine (C) were measured fluorometrically [16]. Glycogen was determined measuring its glucose residues [16]. The values obtained were corrected for the amount of free glucose present at the time of tissue sampling. Oxidative enzymes were represented by citrate synthase (CS), which is responsible for the entry of acetyl coenzyme A (CoA) into the citric acid cycle and 3-hydroxyacyl-CoA dehydrogenase (HAD) which regulates the beta-oxidation of fatty acids. Glycolytic enzymes were represented by hexokinase (HK), which is involved in glucose phosphorylation, phosphofructokinase (PFK), which is a rate limiting enzyme in glycolysis and lactate dehydrogenase $(\mathrm{LDH})$, which is involved in lactate metabolism. To determine maximal enzyme activities, the frozen tissue sample was homogenized in a buffer, containing $\left(\mathrm{mmol} \cdot \mathrm{L}^{-1}\right)$ : sucrose $(25)$, ethylene diamine tetraacetic acid (2), tris-hydroxymethyl-amino methane $(10$, $\mathrm{pH}$ 7.4). CS [17], HAD [18], HK [19], PFK [20] and LDH [21] activities were assessed using a Cobas-BIO autoanalyzer (Roche, Basle, Switzerland). Protein content in the homogenates was measured with the microbicinchoninic acid method of Pierce (Rockford, IL, USA) [22]. For histological examination each biopsy was oriented longitudinally and was rapidly frozen in isopentane. Samples were stored at $-80^{\circ} \mathrm{C}$. Cross sections were cut at $8 \mu \mathrm{m}$ by use of a cryostat kept at $-20^{\circ} \mathrm{C}$. Sections were stained with haematoxylin-eosin and were studied for myopathic changes [23] by a blinded pathologist. The sections were qualitatively scored for presence or absence of the following morphological features: increased numbers of central nuclei, increased variation of muscle fibre diameters, increased numbers of split fibres, increased numbers of vacuoles, increased numbers of abnormal mitochondria, and increased amount of connective tissue.

\section{Pulmonary function tests}

FEV1 and the forced vital capacity were measured (Masterlab; Jaeger, Wurzburg, Germany) until three reproducible recordings were obtained. The highest values were used for analysis. The diffusion capacity of the lung for carbon monoxide (DL,CO) was measured by the singlebreath carbon monoxide method (Masterlab Transfer; Jaeger). Total lung capacity and residual volume were measured by body plethysmography (Masterlab Body; Jaeger). Values were expressed as a percentage of reference values [24]. Blood was drawn from the radial artery while sub-jects were breathing room air. Blood gases were measured using a blood gas analyser (ABL 330; Radiometer, Copenhagen, Denmark).

Anthropometric measures and maximal mouth pressures

Body weight (BW) and height (Ht) were measured standing barefoot. Body mass index (BMI) was calculated 
by dividing BW by $\mathrm{Ht}^{2}$. Fat-free mass (FFM) was determined with a deuterium dilution method [25]. FFM index (FFMI) was calculated by dividing FFM by $\mathrm{Ht}^{2}$. Fat mass (FM) was calculated by subtracting FFM from BW. FM was expressed as a percentage of BW. Respiratory muscle strength was determined by maximal inspiratory and expiratory mouth pressures $(P \mathrm{I}$,max and $P$ E,max, respectively) [26].

\section{Calculations and statistical analysis}

Because protein content tended to be lower in CORT + compared to CORT- patients and HC, muscle enzymatic activity was related to protein content. The total adenine nucleotides (TAN) was calculated by adding ATP, ADP and AMP. Total muscle creatine was calculated by adding $\mathrm{PC}$ and $\mathrm{C}$. On analysis of qualitative morphology the presence of a morphological feature was scored 1 and absence was scored 0 . The total amount of morphological abnormalities was calculated by adding the scores of individual morphological features. Comparisons between groups were performed using the Mann Whitney U-test. Frequency data were compared using the Chi-squared test. Linear regression analysis was used to study relationships between parameters. Significance was determined at the 5\% level. Statistical analyses were performed using the SPSS for Windows statistical package (SPSS, Inc., Chicago, IL, USA).

\section{Results}

\section{Subject characteristics}

Pulmonary function, anthropometric measurements and maximal mouth pressures are listed in table 1. CORT- patients had severe airflow obstruction, marked air trapping, moderate hyperinflation, reduced $D$ L,CO and slightly reduced values of arterial oxygen with, on average, normocapnia. In the $\mathrm{HC}$ group, all lung function parameters were in the normal range. Compared with the $\mathrm{HC}$, in CORT- patients the BMI was significantly lower because of a significantly decreased FFMI, whereas the FM was not different from that of the $\mathrm{HC}$. Both $P \mathrm{I}$,max and $P \mathrm{E}$, max were decreased in CORT- patients compared with HC. No differences in lung function parameters, anthropometric measures and maximal mouth pressures were found between CORT- and CORT+ patients.

\section{Medical treatment}

$\mathrm{CORT}+$ patients were using mean $\pm \mathrm{SD} 7.4 \pm 4.8 \mathrm{mg}$, prednisolone day $^{-1}$ (range: $5-17.5 \mathrm{mg}$ ) as maintenance treatment. They were using this treatment for $4.8 \pm 5.7 \mathrm{yrs}$, (range: $1-20 \mathrm{yrs})$. CORT + and CORT- patients were treated with $2.0 \pm 1.6$ (range: $0-5$ ) and $1.4 \pm 1.1$ (range $0-3$ ) steroid burst regimens in the preceding year, respectively (NS). The patient subgroups did not differ in concomitant treatment.
Table 1. - Characteristics of chronic obstructive pulmonary disease (COPD) patients and healthy controls

\begin{tabular}{lccc}
\hline & Controls & CORT- & CORT + \\
\hline Subjects n & 10 & 15 & 14 \\
Age yrs & $66 \pm 8$ & $65 \pm 6$ & $66 \pm 8$ \\
FEV1 \% & $112 \pm 9$ & $33 \pm 9^{*}$ & $30 \pm 11$ \\
FVC \% & $114 \pm 7$ & $86 \pm 18^{*}$ & $79 \pm 20$ \\
$D \mathrm{~L}, \mathrm{CO} \%$ & $115 \pm 22$ & $58 \pm 26^{*}$ & $57 \pm 36$ \\
$\mathrm{RV} \%$ & $113 \pm 14$ & $202 \pm 60^{*}$ & $209 \pm 78$ \\
$\mathrm{TLC} \%$ & $109 \pm 5$ & $125 \pm 21^{*}$ & $129 \pm 20$ \\
$P \mathrm{a}, \mathrm{O}_{2} \mathrm{kPa}$ & $11.4 \pm 1.5$ & $9.4 \pm 1.0^{*}$ & $9.1 \pm 1.0$ \\
$P \mathrm{a}, \mathrm{CO}_{2} \mathrm{kPa}$ & $4.6 \pm 0.5$ & $5.2 \pm 0.6^{*}$ & $5.6 \pm 0.7$ \\
$\mathrm{BMI} \mathrm{kg} \cdot \mathrm{m}^{-2}$ & $25.9 \pm 2.2$ & $22.1 \pm 4.3^{*}$ & $23.3 \pm 3.0$ \\
$\mathrm{FFMI} \mathrm{kg} \cdot \mathrm{m}^{-2}$ & $19.5 \pm 1.2$ & $15.9 \pm 1.9^{*}$ & $16.2 \pm 1.8$ \\
$\mathrm{FM} \mathrm{\%}$ & $24 \pm 5$ & $27 \pm 9$ & $30 \pm 7$ \\
$P \mathrm{I}, \mathrm{max} \mathrm{cmH}_{2} \mathrm{O}$ & $-102 \pm 21$ & $-74 \pm 23^{*}$ & $-69 \pm 24$ \\
$P \mathrm{E}, \mathrm{max} \mathrm{cmH}_{2} \mathrm{O}$ & $101 \pm 19$ & $83 \pm 17^{*}$ & $85 \pm 19$ \\
\hline
\end{tabular}

Data are presented as mean \pm SD. CORT-: COPD patients not using prednisolone; CORT+: COPD patients using prednisolone; FEV1: forced expiratory volume in one second; FVC: forced vital capacity; $D \mathrm{~L}, \mathrm{CO}$ : diffusion capacity of the lung for carbon monoxide; RV: residual volume; TLC: total lung capacity; $P \mathrm{a}, \mathrm{O}_{2}$ : oxygen tension in arterial blood; $\mathrm{Pa}_{\mathrm{a}}, \mathrm{CO}_{2}$ : carbon dioxide tension in arterial blood; BMI: body mass index; FFMI: fat-free mass index; FM: fat mass as a percentage of body weight; PI,max: maximal inspiratory mouth pressure; $P$ E,max: maximal expiratory mouth pressure. FFMI and FM were measured in eight controls, 10 CORT- and $10 \mathrm{CORT}+$ patients. $*: \mathrm{p}<0.05$ compared to controls.

\section{High energy phosphates and related compounds}

Values of muscle metabolites are summarized in table 2. No significant differences were found between the CORTpatient subgroup and the HC group in ATP, ADP, AMP, or TAN. However, CORT-patients had significantly lower ATP/ADP ratios compared to the HC group. IMP could be detected in 13 of 15 CORT- patients compared with three of $10 \mathrm{HC}$ (Chi-squared=8.4, $\mathrm{p}=0.004)$. The mean IMP level

Table 2. - Muscle high energy phosphates and related compounds

\begin{tabular}{lccc}
\hline & Controls & CORT- & CORT + \\
\hline $\mathrm{n}$ & 10 & 15 & 14 \\
ATP & $19.2 \pm 3.5$ & $18.7 \pm 2.5$ & $16.6(3.5)$ \\
$\mathrm{ADP}$ & $3.1 \pm 0.5$ & $3.3 \pm 0.5$ & $3.0(0.6)$ \\
$\mathrm{AMP}$ & $0.09 \pm 0.03$ & $0.12 \pm 0.03$ & $0.10(0.03)$ \\
$\mathrm{ATP} / \mathrm{ADP}$ & $6.2 \pm 0.6$ & $5.7 \pm 0.5^{*}$ & $5.6(0.5)$ \\
$\mathrm{TAN}$ & $22.4 \pm 3.9$ & $22.2 \pm 3.0$ & $19.7(4.1)$ \\
$\mathrm{PC}$ & $72 \pm 10$ & $80 \pm 13$ & $76(19)$ \\
$\mathrm{C}$ & $36 \pm 7$ & $45 \pm 8^{*}$ & $42(10)$ \\
$\mathrm{PC} / \mathrm{C}$ & $2.0 \pm 0.2$ & $1.8 \pm 0.2$ & $1.8(0.4)$ \\
Ctot & $108 \pm 16$ & $125 \pm 20$ & $118(27)$ \\
IMP yes/no & $3 / 7$ & $13 / 2^{*}$ & $11 / 3$ \\
IMP & $0.08 \pm 0.02$ & $0.14 \pm 0.08^{*}$ & $0.16 \pm 0.13$ \\
Glycogen & $286 \pm 88$ & $268 \pm 64$ & $249 \pm 60$ \\
\hline
\end{tabular}

Values are presented as mean $\pm \mathrm{SD}$ and are expressed as mmol $\cdot \mathrm{kg}^{-1}$ dry weight. CORT-: chronic obstructive pulmonary disease (COPD) patients not using prednisolone; CORT+: COPD patients using prednisolone; ATP: adenosine triphosphate; ADP: adenosine diphosphate; AMP: adenosine monophosphate; TAN: ATP+ADP+AMP; PC: phosphocreatine; $\mathrm{C}$ : creatine; Ctot: PC+ $\mathrm{C}$; IMP: inosine monophosphate. Glycogen is expressed as mmol glycosyl units $\mathrm{kg}^{-1}$ dry weight. $*$ : $\mathrm{p}<0.05$ compared to controls. 
Table 3. - Muscle enzyme capacities related to muscle protein content

\begin{tabular}{lccc}
\hline & Controls & CORT- & CORT+ \\
\hline $\mathrm{n}$ & 10 & 14 & 12 \\
$\mathrm{CS}$ & $58 \pm 14$ & $69 \pm 16$ & $71 \pm 17$ \\
$\mathrm{HAD}$ & $38 \pm 12$ & $41 \pm 19$ & $40 \pm 17$ \\
$\mathrm{HK}$ & $3.3 \pm 1.8$ & $3.0 \pm 1.5$ & $3.1 \pm 1.3$ \\
$\mathrm{PFK}$ & $242 \pm 114$ & $274 \pm 85$ & $260 \pm 161$ \\
$\mathrm{LDH}$ & $465 \pm 218$ & $464 \pm 173$ & $413 \pm 181$ \\
Protein & $151 \pm 30$ & $141 \pm 31$ & $128 \pm 35$ \\
\hline
\end{tabular}

Data are presented as mean \pm SD in U.g protein ${ }^{-1}$. CORT-: chronic obstructive pulmonary disease (COPD) patients not using prednisolone; CORT+: COPD patients using prednisolone; CS: citrate synthase; HAD: 3-hydroxyacyl-coenzyme A dehydrogenase; HK: hexokinase; PFK: phosphofructokinase; LDH: lactate dehydrogenase. Protein is expressed as $\mathrm{mg} \cdot \mathrm{g}^{-1}$ wet weight.

in the 13 CORT- patients with detectable IMP was $0.14 \pm 0.08$, ranging $0.06-0.29 \mathrm{mmol} \cdot \mathrm{kg}^{-1}$ dry weight. The mean IMP level of the three $\mathrm{HC}$ with detectable IMP was $0.08 \pm 0.02$, ranging $0.06-0.09 \mathrm{mmol} \cdot \mathrm{kg}^{-1}$ dry weight. In CORT- patients with detectable IMP a significant correlation was found between IMP levels and the ATP/ADP ratio $(\mathrm{r}=-0.72, \mathrm{p}=0.006)$. $\mathrm{C}$ was significantly higher in CORT- patients compared to HC. Comparing CORT- and CORT + patient subgroups, no significant differences were found in muscle high energy phosphates or glycogen.

\section{Muscle metabolic enzyme capacity}

Muscle enzyme capacities are listed in table 3 . No significant differences in oxidative or glycolytic enzyme capacities were found between the CORT- patient subgroup and the HC group. In CORT- patients and $\mathrm{HC}$ a positive correlation was found between CS and HAD enzyme capacities $(r=0.68, p=0.001)$. No significant relationships were found between enzyme capacities and muscle high

Table 4. - Abnormalities in qualitative muscle morphology

\begin{tabular}{lccc}
\hline & Controls & CORT- & CORT + \\
\hline $\mathrm{n}$ & 10 & 14 & 11 \\
Increased numbers of & 60 & 36 & 46 \\
$\quad$ central nuclei & 30 & 27 & 9 \\
$\quad \begin{array}{l}\text { Increased variation in muscle } \\
\text { fibre diameters }\end{array}$ & & & \\
$\quad \begin{array}{l}\text { Increased numbers of split } \\
\text { fibres }\end{array}$ & 10 & 7 & 9 \\
Increased numbers of vacuoles & 10 & 0 & 0 \\
Increased numbers of abnormal & 0 & 0 & 0 \\
mitochondria & & & \\
Increased amount of connective & 0 & 0 & 0 \\
tissue & & & \\
Sum of all myopathic features & 30 & 57 & 55 \\
0 & 30 & 29 & 27 \\
1 & 40 & 7 & 18 \\
2 & 0 & 7 & 0 \\
3
\end{tabular}

Data are presented as percentages. CORT-: chronic obstructive pulmonary disease (COPD) patients not using prednisolone; CORT+: COPD patients using prednisolone. energy phosphate contents. Comparing CORT- and CORT + patient subgroups no differences were found in muscle enzyme capacities.

\section{Qualitative morphology in muscle fibres}

Qualitative morphological changes are listed in table 4. In all of the subjects the sum of the morphological abnormalities varied between $0-3$. No significant differences in the occurrence of individual morphological changes reflecting myopathic alterations, or in the sum of all morphological changes, were found between the CORT- patient subgroup and the HC group. Also comparing CORT- and CORT+ patient subgroups, no differences in qualitative muscle morphology were found.

\section{Discussion}

In the present study, in anterior tibialis muscle, oxidative and glycolytic enzyme capacities were not different in CORT- patients and HC. These findings are in accordance with GEA et al. [27], who did not find abnormalities in enzyme capacities in deltoid muscle. However, in studies examining quadriceps femoris muscle, a decreased oxidative capacity as measured by $\mathrm{CS}[3,6]$ and HAD [6] and an increased glycolytic capacity as measured by PFK [6] were found. With comparable patient characteristics, other factors seem to be more important in causing the discrepancies between the studies. In the above-mentioned studies, three different muscles were investigated, which differ greatly in structure and function, tibialis anterior muscle being primarily a postural muscle containing a high percentage of slow, type I muscle fibres $(70 \%)$, deltoid muscle having both tonic and phasic activity and containing 50-60\% type I muscle fibres, and quadriceps femoris muscle being primarily a locomotor muscle containing only $40 \%$ type I muscle fibres [28].

In COPD patients both systemic factors such as hypoxia and nutritional depletion and local factors, such as activity level might influence muscle enzyme capacities. At present it is unknown to what extent muscles of different fibre type distribution and function are affected by the combination of these factors. As for the latter factor, it is known that complete immobilization causes a greater decrease in oxidative capacity in predominantly type I muscles, compared to predominantly type II muscles [29]. On the other hand it is probable that the relative inactivity (but not complete immobilization) that frequently occurs in severe COPD patients affects a locomotor muscle to a greater extent than a nonlocomotor muscle. This latter phenomenon might explain the fact that decreased oxidative capacity has been found in quadriceps femoris muscle, but not in deltoid or tibialis anterior muscle of COPD patients.

In CORT- patients compared with the HC group elevated IMP levels and decreased ATP/ADP ratios were found in resting anterior tibialis muscle. Furthermore, IMP levels were inversely related with ATP/ADP ratios. These findings have been reported earlier in a larger patient group [4]. Based on these findings, it was hypothesized that in COPD patients, an imbalance in ATP utilization and resynthesis already exists in resting anterior tibialis muscle. It is noteworthy that signs of imbalance in ATP resynthesis and utilization can be found, without accompanying changes in the capacities of the most important oxidative 
and glycolytic enzymes. This suggests that other factors play a role in the observed disturbances in muscle high energy phosphate metabolism.

The effect of low dose maintenance treatment with oral prednisolone on parameters of muscle energy metabolism was evaluated comparing CORT- and CORT + patient subgroups. No differences in muscle high energy phosphate levels were found. Based on electron microscopic studies reporting structural changes in mitochondria, it was hypothesized that oxidative metabolism might be impaired during use of corticosteroids. However, in the present study no differences in oxidative and glycolytic enzyme capacities were found between patient subgroups. In accordance with these findings, in rat muscle, no alterations of CS and PFK capacity were found during prolonged use of prednisolone [30]. In contrast to the current findings, in patients with rheumatoid arthritis using long-term prednisolone $(7.5-10 \mathrm{mg})$, decreased CS and HAD capacities were found in quadriceps femoris muscle [31]. However, in the quoted latter study only $37.5 \%$ type I fibres were found. Because it is unlikely that this decrease was solely caused by the use of corticosteroids, this finding suggests that other factors, such as inactivity might have contributed.

In most experimental studies evaluating corticosteroid induced changes in muscle metabolism, elevated muscle glycogen levels were found $[9,10,30]$. This finding has been confirmed in a human study by FERNANDEZ-SOLA et al. [11], examining quadriceps femoris muscle of patients with bronchial asthma on chronic treatment with prednisolone (mean daily dose $17.3 \mathrm{mg}$ ). Therefore, it is notable that in the present study, no differences in muscle glycogen levels were found comparing CORT- and CORT+ patients.

Discrepancies between the above mentioned studies and the current study might have been caused by the fact that in the former studies higher dosages of corticosteroids were used. Furthermore, in experimental studies steroidinduced changes were found to be more extensive in type II compared to type I muscle. Therefore, the effect of corticosteroids on anterior tibialis muscle might be less than expected in muscles containing more type II fibres.

In agreement with FERNANDEZ-SOLA et al. [11], but in contrast to DeCRAMER et al. [12], no differences in parameters of qualitative muscle morphology were found between CORT- and CORT + patients in the present study. Although comparable qualitative morphological features were assessed, the current study cannot readily be compared with the latter study, in which a higher dose of corticosteroids was used (14.2 mg methylprednisolone) and a predominantly type II muscle was analysed. Furthermore, the subgroup of patients in the study by DECRAMER et al. [12] that showed myopathic morphological features in the muscle biopsy, suffered from severe skeletal and respiratory muscle weakness. Unfortunately, in the present study, skeletal muscle function was not assessed. However, mouth pressures were not different between the patient subgroups.

In the present study, some morphological abnormalities were also found in HC. Because an increased number of central nuclei can also be found near a myotendinous junction [23], it could be speculated that this phenomenon be partly explained by the location of the sample site. However, because care was taken to obtain biopsies from the belly of the muscle and because no associated increased amount of fibre splitting was present, this explanation seems improbable.

In conclusion, in tibialis anterior muscle of stable chronic obstructive pulmonary disease patients, no alterations in oxidative and glycolytic enzyme capacities were found, despite the fact that indications were found for an imbalance between the utilization and resynthesis of muscle adenosine triphosphate. Furthermore, a comparison between chronic obstructive pulmonary disease patients who never used maintenance treatment with glucocorticosteroids, and chronic obstructive pulmonary disease patients using maintenance treatment with low dose prednisolone showed no effect of this treatment on parameters of muscle energy metabolism and on qualitative muscle morphology.

\section{References}

1. Gosselink R, Troosters T, Decramer M. Peripheral muscle weakness contributes to exercise limitation in COPD. $\mathrm{Am}$ J Respir Crit Care Med 1996; 153: 976-980.

2. Bernard S, Leblanc P, Whittom F, et al. Peripheral muscle weakness in patients with chronic obstructive pulmonary disease. Am J Respir Crit Care Med 1998; 158: 629-634.

3. Maltais F, Simard A, Simard C, Jobin J, Desgagnes P, Leblanc P. Oxidative capacity of the skeletal muscle and lactic acid kinetics during exercise in normal subjects and in patients with COPD. Am J Respir Crit Care Med 1996; 153: 288-293.

4. Pouw EM, Schols AMW, van der Vusse GJ, Wouters EFM. Elevated inosine monophosphate levels in resting muscle of patients with stable chronic obstructive pulmonary disease. Am J Respir Crit Care Med 1998; 157: 453457.

5. American Thoracic Society/European Respiratory Society. Skeletal muscle dysfunction in chronic obstructive pulmonary disease. Am J Respir Crit Care Med 1999; 156: S1-S40.

6. Jakobsson P, Jorfeldt L, Hendriksson J. Metabolic enzyme activity in the quadriceps femoris muscle in patients with severe chronic obstructive pulmonary disease. Am J Respir Crit Care Med 1995; 151: 374-377.

7. Kimberg DV, Loud AV, Wiener J. Cortisone-induced alterations in mitochondrial function and structure. J Cell Biol 1968; 37: 63-79.

8. Engel AG. Electron microscopic observations in thyrotoxic and corticosteroid-induced myopathies. Mayo Clin Proc 1966; 41: 785-796.

9. Kerpolla W. Inhibition of phosphorylase with cortisone and its activation with adrenaline in rabbits. Endocrinology 1952; 51: 192-202.

10. Shoji S, Takagi A, Sugita H, Toyokura Y. Muscle glycogen metabolism in steroid-induced myopathy of rabbits. Exp Neur 1974; 45: 1-7.

11. Fernandez-Sola J, Cusso R, Picado C, Vernet M, Grau JM, Urbano-Marquez A. Patients with chronic glucocorticoid treatment develop changes in muscle glycogen metabolism. J Neurol Sci 1993; 117: 103-106.

12. Decramer M, Bock de V, Dom R. Functional and histologic picture of steroid-induced myopathy in chronic obstructive pulmonary disease. Am J Respir Crit Care Med 1996; 153: 1958-1964.

13. American Thoracic Society. Standards for the diagnosis and care of patients with chronic obstructive pulmonary disease (COPD). Am J Respir Crit Care Med 1995; 152: S77-S120. 
14. Dietrichson P, Coackley P, Smith PEM, Griffiths RD, Helliwell TR, Tedwards RH. Conchotome and needle percutaneous biopsy of skeletal muscle. J Neur Neurosurg Psych 1987; 50: 1461-1467.

15. Van der Vusse GJ, Jansen GEM, Coumans WA, Kuipers H, Does RJMM, ten Hoor F. Effect of training and 15-, 25-, and $42-\mathrm{km}$ contests on the skeletal muscle content of adenine and guanine nucleotides, creatine phosphate, and glycogen. Int J Sports Med 1989; 10: Suppl. 3, S146-S152.

16. Van der Vusse GJ, Coumans WA, van der Veen FH, Drake A, Flameng W, Suy R. ATP, creatine phosphate and glycogen content in human myocardial biopsies: markers for the efficacy of cardioprotection during aortocoronairy bypass surgery. Vasc Surg 1984; 18: 127-134.

17. Shephard D, Garland PB. Citrate synthase from rat liver. Methods Enzymol 1969; 13: 11-16.

18. Bradshaw RA, Noyes BE. L-3-hydroxyacyl Coenzyme A dehydrogenase from pig heart muscle. Methods Enzymol 1975; 35: 122-128.

19. Sols A, De La Fuente G, Villar-Palasi C, Asensio C. Substrate specificity and some other properties of bakers yeast hexokinase. Methods Enzymonol 1958; 30: 92- 101.

20. Ling KH, Paetkau V, Marcus F, Lardy HA. Phosphofructokinase. Methods Enzymonol 1966; 9: 425-429.

21. Bergmeyer HU, Bernt E. Lactate dehydrogenase. In: Bergmeyer HU, ed. Methods of enzymatic analysis. Vol. 2. New York, London, Weinheim Verlag Chemie GmbH, Academic Press, Inc., 1974; pp. 575-579.

22. Smith PK, Krohn RI, Hermanson GT. et al. Measurement of protein using bicinchoninic acid. Anal Biochem. 1995; 150: $163-279$.
23. Dubowitz V, Sewry CA, Fitzimons RB. Definition of pathological changes seen in muscle biopsies. In: Dubowitz V, ed. Muscle biopsy: a practical approach. 2nd Edn. London, UK, Balliere Tindall Publishers, pp. 82-128.

24. Quanjer PH. Standardized lung function testing. Bull Eur Physiopathol Respir 1983; 19: 7-44.

25. Westerterp KR, Wouters L, van Marken Lichtenbelt WD. The Maastricht protocol for the measurement of body composition and energy expenditure with labelled water. Obes Res 1995; 3: Suppl. 1, 49-57.

26. Black LF, Hyatt RE. Maximal respiratory pressures: normal values and relationship to age and sex. Am Rev Respir Dis 1969; 99: 696-702.

27. Gea JG, Pasto M, Blanco L, et al. Biomolecular metabolism in a peripheral skeletal muscle of the upper limbs in COPD patients. Am J Respir Crit Care Med 1999; 159: A579.

28. Johnson MA, Polgar J, Weightman D, Appleton D. Data on the distribution of fibre types in thirty-six human muscles. J Neurol Sci 1973; 18: 111-129.

29. Appell HJ. Muscular atrophy following immobilisation. A review. Sports Med 1990; 10: 42-58.

30. Koerts-de Lang E, Hesselink MKC, Drost MR, van der Vusse GJ, Wouters EFM, Schols AMWJ. Enzyme activity of rat tibialis anterior muscle differs between triamcinolone and prednisolone treatment and nutritional deprivation. Eur J Appl Physiol 1999; 79: 274-279.

31. Danniskiold-Samsoe B, Grimby G. The influence of prednisolone on the muscle morphology and muscle enzymes in patients with rheumatoid arthritis. Clin Sci 1986; 71: 693-701. 